respiration were strong and rapid and the heart sounds were very loud. The boy was unable to speak. I found the suspected bottles to contain glycerine of belladonna. I ascertained that the boy had not vomited. As the evidences of belladonna poisoning were undoubted $I$ washed out the stomach with the syphon. A good deal of the water first introduced was vomited over the child's nightshirt, which was of a dark brown colour, so that it was difficult to tell from its appearance whether it contained belladonna extract or not. That which came through the syphon was clear. There was no odour of belladonna from the washings. Considering that five hours bad elapsed since the poison was taken I deemed it highly improbable that any of it still remained in the stomach.

After completing the washing of the stomach I gave a hypodermic injection of a quarter grain of morphia sulphate and a tablespoonful of castor-oil by the mouth, which was swallowed. In a short time the boy became quiet and I left the house, having directed the parents to send for me should any further alarming symptoms arise.

I was unable to see the patient again until $11 \mathrm{~A} . \mathrm{M}$. Then I ascertained that he had slept well till 10 , and on awaking still exhibited symptoms of the same kind, but less aggravated. The delirium was now of a hilarious character, somewhat resembling drunkenness. The child laughed and chatted about his school experiences, games, fights, \&c. The bowels had not yet moved. I then gave another hypodermic injection of one-quarter grain of morphia sulphate and three grains of calomel by the mouth. The boy did not again sleep till about 11 o'clock. During the whole of that day somebody had to remain constantly at the bedside to keep him in bed. Several times he attempted to bite those trying to control his movements. Although the mouth was very dry and the tongue was constantly rolled about the boy refused food or drink and spat out whatever was introduced into his mouth. At two in the afternoon, the bowels not having yet moved, the mother gave a small soap-and-water enema, after which there were three or four liquid motions. The boy continued in the same mental state till about 11 P.M., when he fell asleep. Shortly after 11 P. M. I visited the patient, and finding him sleeping tranquilly I judged it best to do nothing. He had a good night's rest. The following morning at $10 \mathrm{I}$ found him awake and presenting no abnormal symptoms except dilatation of the pupils which persisted for four days. No rash at any time appeared upon the body and there was no peeling of the skin. After recovery the boy seemed to have no recollection of the incidents which occurred during his illness.

This case may be regarded as illustrating two points, the one necessarily inversely as the other: (1) the tolerance of the child for belladonna; and (2) the efficiency of morphia as an antidote. 1. The dose swallowed was certainly a large one. The chief alkaloid in belladonna is now believed to be hyoscyamine, not atropine. These alkaloids are isomeric and it is considered probable that atropine is a conversion product of hyoscyamine. Extract of belladonna is estimated to contain about 1 per cent. of alkaloid. The glycerine contains 50 per cent. of the extract. In this instance fully half an ounce of the glycerine or two drachws of the extract were taken, representing about one grain of alkaloid. The medicinal dose of both alkaloids is from $\frac{1}{10}$ th grain to soth grain. The fact that the patient remained untreated for five hours without exhibiting the extreme symptoms of poisoning by the drug points to a remarkable tolerance. Taylor quotes as remarkable a case in which a woman recovered after swallowing three drachms of the extract; but although emetics were administered successfully she did not completely recover until tive weeks had elapsed. In the case under discussion, when we consider the enormous dose and the great probability that it was all absorbed, it would seem that tolerance alone can hardly explain the speedy recovery. 2. It is here interesting to note that "the Elinburgh committee, presided over by Dr. Hughes Bennett, conclude : (1) that sulphate of atropia is, within a limited range, physiologically antagonistic to meconate of morphia; (2) that meconate of morphia does not act antidotally after a large dose of atropia, and thus, while atropia is an antidote to morphia, morphia is not an antidote to atropia." Physostigmine, in relation to atropine, is considered to have a wider range of antidotal action than morphia. "Although it is experimentally proved that atropine can avert death from physostigmine the question yet remains unsolved whether physostigmine can prevent death from atropine." The above two quotations from
Ringer and $S$ tinsbury make it appear that atropine is a good servant, but a bad master.

Those who advocate morphia as an antidote to atropine recommend a quarter of a grain of the former to one-thirtieth of a grain of the latter. It is conceivable that in the child, where there is tolerance of the one and intolerance of the other, the proportion of morphia to atropine ought to be much less. In the light ot the more modern view that hyoscyamine is the chief alkaloid in belladonna, these conclusions regarding atropine are irrelevant where belladonna is the poison under consideration, unless we assume that atropine and hyoscyamine bave identical physiological action. Such an assumption stands in need of investigation, as indeed does the whole subject, for the opinions to be found in onr text-books as to the alkaloids of belladonna and hyoscyamus, their antidotes, and their posology are very conflicting. The fact that in this case such large doses of morphia produced in the child so little of the usual effects of the drug is not so significant as it would at first sight appear, for it merely confirms, what is generally admitted, that belladonna antagonises morphia. The case for the converse proposition must rest entirely upon the non-appearance of the gravest symptoms of belladonna poisoning and the rapid recovery from those symptoms which did appear.

Auchinleck, Ayrshire.

\section{DERMATITIS FROM ARSENIC IN STOCKINGS.}

By F. W. TUNNICLIFFE, M.D., M.R.C.P. LOND.,

PROFHSSOR OF MATERIA MEDICA AND PHARMACOLOGY IN KING's COLLEGE, LONDON ;

AND

OTTO ROSENHEIM, PH.D., F.C.S.

THAT dyed materials when brought into contact with the skin may give rise to local irritation, occasionally culminating in actual dermatitis, has long been known to dermatologists. From the many cases available for quotation we need only refer here to those recorded in THE LANCET by Mr. Henry Lee. ${ }^{1}$ In the early period of aniline dye manufacture the effect of dyed stockings in this direction was easily traceable to the large percentage of arsenic acid used in the preparation of these dyes (fuchsine red, \&c.). That the external application of arsenic is productive of severe and even fatal poisoning is well established, as may be seen upon reference to the text-books of toxicology. These considerations, confirmed by many other observations, resulted in Germany in the passing of a law prohibiting the use of any dye stuff containing arsenic for the purpose of colouring food, wearing apparel, or domestic objects. ${ }^{2}$ Since the passing of this law practically all the aniline colours placed on the market have been free from arsenic. A knowledge of these facts rendered the etiology of the following cases which have recently been brought under our notice at first sight somewhat obscure. We were, however, able clearly to trace them to the presence of arsenic in the stockings worn.

Before entering into the subject further, we think it best to give a short description of the cases in question. For the medical notes of the first case we are indebted to Dr. J. Cohn.

CASE 1.- "A boy, nine years old, complained on March 3rd last of pain in the right leg. Examination showed the presence of an inflammatory nodule of about the size of a walnut, situated at the junction of the upper with the middle third of the calf. The nodule was regarded as being probably a commencing furuncle, and sedative lotions and compresses were applied. Upon seeing the patient on the next morning each leg was found to be covered with from 10 to 12 nodules. The nodules were the same as the one observed on the previous day, except somewhat smaller, and were situated between the foot and the knee, stopping abruptly short where the stockings ceased to be in contact with the skin. This directed my attention to the stockings and upon inquiry it was found that the boy had put on a 1887; Erläuterungen zu dem Gesetzentwurf, \&c. 
new pair of stockings on the same morning as the first nodule appeared. In the evening of the same day (viz., the second day of the affection) the boy was again seen. He was restless and since the last visit had vomited, without any apparent cause. His condition, however, did not seem serious, the temperature being normal and the pulse only slightly accelerated. There was no diarrhoea. The case caused no further anxiety but necessitated the boy being kept in bed for three days. The nodules gradually faded and on the fifth day the boy went out. On this day he was able to put on his stockings and wore one of bis old pairs. On the eighth day, however, he put on the same stockings that seemed to have been the cause of the original irriration. They had been carefally washed in the meantime with soda. On the evening of the same day another crop of nodules appeared which faded in the course of a few days under similar treatment and the entire discarding of the stockings in question."

CASE 2.-This case, which was much less severe, may be described as follows. The patient, a female, noticed a marked tenderness of the feet, especially the soles, after wearing for a few hours a pair of new black stockings. Although no gross local inflammation was produced the pain upon pressure was so intense that she conld not stand or walk upon her feet. The stockings were discarded and the symptoms disappeared. A few days afterwards the same stockings, which had been washed in the meantime, were again worn with a recurrence of the symptoms, these latter again disappearing upon leaving off the stockings.

The clinical history of these cases suggests strongly the presence of sume chemical irritant in the stockings. In Case 1 we were able to obtain for chemical examination (1) a pair of new stockings identical with those the wearing of which was followed by the dermatitis; and (2) the stockings actually worn by the boy after they had been twice washed. In Case 2 we obtained an unworn pair of stockings from the same lot as those which had caused the trouble. The stock. ings of the patient in Case 1 were long, black, machine-made woollen stockings, weighing 120 grammes; a piece of 100 square centimetres weighed five grammes. The colouring matter proved on examination to be a chrome black. The ash contained chromium and the material gave the reactions of logwood. Probably some other yellow colouring matter (fustic, \&c.) was present. On further examination it was found that the stockings contained a considerable quantity of arsenic. Ten grammes treated by Reinsch's method gave a copions sublimate of large white arsenic crystals, and the residual liquid when submitted to Marsh's test gave still a strong black arsenical mirror. A further important result of the qualitative examination was that some of the arsenic was found to be present in a soluble form, since an aqueous extract of the stockings made at the temperature of the water bath, and subsequently subjected to Marsh's test, gave a strong arsenical mirror. In the case of these stockings we estimated the total amount of arsenic and also the amount soluble in water. These estimations were made by distilling with sulphate of iron and ammonia in a hydrochloric acid current, ${ }^{3}$ and final weighing as pyro-magnesium-arsenate. It was found that one pair of stockings contained as much as 75 milligrammes, or 1.2 grains, of arsenic $\left(\mathrm{As}_{4} \mathrm{O}_{\epsilon}\right)$; of this 10.5 milligrammes, or one-sixth of a grain, were soluble in water. A piece having an area of 100 square centimetres contained $3 \cdot 1$ milligrammes of $\mathrm{As}_{4} \mathrm{O}_{6}$. It was further found that the stockings which had been washed twice still contained some soluble arsenic, the watery extract giving a distinct arsenical mirror. After the stockings had been completely extracted with distilled water they still contained 64 milligrammes of $\mathrm{As}_{4} \mathrm{O}_{3}$. The stockings of the patient in Case 2 were only examined qualitatively. The mirrors obtained from both the stockings themselves and the aqueous extract of them were approximately of the same intensity as those derived under similar conditions from the new stockings of the patient in Oase 1.

From the above results we think that the previously obscure etiology of these two skin affections is cleared up and that we may regard them as being caused by the arsenic contained in the stockings, and probably by the soluble moiety. The ordinary stockings worn by the boy in Case 1 in the time between the two attacks were found to be free from arsenic. It must, of course, be remembered in

3 Compare Classen and Ludwig: Bericht der Deutschen Chemischen Gesellschaft, Band xviii, p. 1110 . this connexion that the term "soluble" and "insoluble" with regard to the arsenic content of the stockings is only relative, in that the stockings while being worn would be subjected to the action of an acid secretionnamely, the sweat-by the agency of which some of the insoluble arsenical compounds might be rendered soluble. be fact that the amount of sweat secreted, especially by the feet, is very considerable, is shown by the researches of Cramer, ${ }^{4}$ who found that socks in a given time absorbed their own weight of sweat. In this case the conclusion that some arsenic was absorbed through the skin seems justified, in that we must regard the vomiting as produced by the excretion by the stomach of arsenic absorbed by the skin. 1 his same phenomenon has been recorded by other observers.

We have heard incidentally of other cases of dermatitis which, in the light of our present knowledge, we should regard as having a similar eticlogy to the above. Unfortunately these have not been observed with sufficient exactitude, nor was the material in question available for chemical examination. The indications, however, were sufficient to induce us to examine this matter further, with the view of ascertaining to what extent ordinary dyed goods contained arsenic.

We first examined three samples of black woollen stockings purchased at varions prices in different parts of London, all emanating from the same wholesale firm and from the one which supplied the original stockings worn by the patient in Case 1. They all contained arsenic, but apparently in smaller amount than the stockings pr ductive of the dermatitis. We next obtained one sample from each of four other makers, and we found that arsenic was present in each case to a larger or a smaller degree. Three samples of " $\tan$ " mixed wool and silk socks were contaminated as well. We examined also a pair of ordinary woollen gloves and found them to be arsenicated. This had especial interest in view of the case quoted by Sell ${ }^{5}$ in which a severe dermatitis of the hands was produced by the wearing of a pair of marine-blue gloves. A sample of black loose wool was found to be free from arsenic.

Upon inquiring into the source of the arsenic in the goods examined by us we found that arsenic in various forms is used in the process of dyeing. In the standard work on dyeing by Knecht and Kawson ${ }^{6}$ it is stated that a solution of white arsenic in acetic acid to which some glycerine has been added is used and called " purple fixing colour." The anthors also say ${ }^{7}$ that " sodium arseniate is extensively used as a fixing agent for mordants. In Jarn dyeing it affects the hands of the workmen owing to its poisonous properties. As it is so dangerous ...... its use is objectionable." Another possible source, although more unlikely, would be the use of highly arsenicated sulphuric acid in the preparation of the chromic acid mordant bath.

The use of arsenical mordants has been recognised to some extent by the German law quoted above. Their use is allowed upon two conditions-namely, that no soluble compound of arsenic be present in the finished article, and that the total quantity of arsenic be always less than two milligrammes per 100 square centimetres. ${ }^{8}$ A similar law exists in Austria. In Sweden the law is much more stringent. The materials used for the manufacture of wearing apparel, \&c., must not contain arsenic to such an extent as to allow 100 square centimetres of the finished article to produce when treated according to a certain test an opaque arsenical mirror. The maximum limit, according to this test, corresponds to about 0.07 milligramme per 100 square centimetres.

It will be seen that the stockings which caused the derma it is in our two cases contained arsenic considerably in excess of the amount allowed by the above-named countries, and although no doubt individuals differ largely inter se with regard to their sensitiveness to arsenic it would seem that if arsenical mordants cannot be dispensed with, their use should at least be supervised. The presence of soluble arsenical compounds in those materials which come into intimate contact with the skin can certainly be avoided, and should be prohibited.

4 Quoted from Prausnitz: Grundziige der Hygiene, München, 1899, p. 141

\section{Loc. cit} 1. Dy eing, p. 276.

Ioid., p. 195.

8 In this connexion it is interesting to know that a pair of black silk socks as d a pair of yellow stockings obtained from Germany were found to be free from arsenic. 